\title{
Restrictive domain motion in polarization switching of lithium niobate
}

\author{
L.-H. Peng, ${ }^{\text {a) }}$ Y.-J. Shih, and Y.-C. Zhang \\ Department of Electrical Engineering and Institute of Electro-Optical Engineering, \\ National Taiwan University, Taipei, Taiwan, Republic of China
}

(Received 4 April 2002; accepted for publication 5 July 2002)

\begin{abstract}
We investigate the electrostatic control of domain nucleation and motion for periodical polarization $\left(P_{s}\right)$ switching on Z-cut congruent-grown lithium niobate $\left(\mathrm{LiNbO}_{3}\right)$. A tenfold reduction in the tangential field $\left(E_{x}\right)$ can be resolved as the perturbed, positive charge density intervening otherwise the single-domain crystal exceeds $10^{-3} P_{s}$. This concept is realized in a two-step procedure consisting of a high-temperature treatment followed by pulsed field poling. Bulk periodic polarization switching is then realized underneath the oxidized electrode with the lateral $180^{\circ}$ domain motion confined to the edge of the charged domain boundary. The forward domain growth can extend through the 500- $\mu$ m-thick substrate and reach an aspect ratio as large as 150 . These observations are attributed to the constraint on domain nucleation and the suppression of $E_{x}$ by the positive charge originating from the divergence of $P_{s}$ at the inverted domain boundary near the subsurface regime. (C) 2002 American Institute of Physics. [DOI: 10.1063/1.1503169]
\end{abstract}

The use of a quasi-phase-matching (QPM) technique ${ }^{1}$ in nonlinear wave interactions has been actively pursued in the field of nonlinear optics. In ferroelectric nonlinear crystals, the QPM condition can be fulfilled by periodical domain reversal at every coherent length $l_{c}=\lambda_{\omega} / 4\left(n_{2 \omega}-n_{\omega}\right)$ such that the sign change in the spontaneous polarization $\left(P_{s}\right)$, and therefore the second-order nonlinear susceptibility $\chi^{(2)}$, can be used to compensate destructive phase interference. ${ }^{2}$ Efficient parametric processes, ${ }^{3}$ optical and acoustic applications, ${ }^{4}$ just to name a few, are vivid examples benefiting from such investigations. Such a plethora of research activities are ascribed to the availability of QPM structures of periodically poled lithium niobate (PPLN) $;{ }^{5}$ lithium tantalate (PPLT); ${ }^{6}$ potassium titanyl phosphate (PPKTP), ${ }^{7}$ and its isomorphic family; ${ }^{8}$ and more recently, the commercialization of these materials. A common practice is to invoke chemical exchange in the fabrication of QPM structures. Albeit simple in nature, employment of such methods inevitably results in surface phases of $\mathrm{H}_{x} \mathrm{Li}_{1-x} \mathrm{NbO}_{3}$ (Ref. 9) and $\mathrm{H}_{x} \mathrm{Li}_{1-x} \mathrm{TaO}_{3}$ (Ref. 10) or introduces surface refractive index dispersion in ion-changed $\mathrm{KTP},{ }^{11}$ which are known to be detrimental to conversion efficiency.

Pulsed field poling, on the other hand, offers an alternative domain reversal approach by delivering a pulsed field across the crystal's polar $(Z)$ axis to overcome the coercive field $\left(E_{c}\right)$ and initiate the polarization switching process. ${ }^{12}$ Based upon early studies of $\mathrm{BaTiO}_{3}$, it is suggested that the surface nucleation rate, sidewise expansion, and coalescence of the domain constitute three major steps in the kinetics of polarization switching. ${ }^{13,14}$ The situation, however, becomes complicated on congruent-grown $\mathrm{LiNbO}_{3}$ and $\mathrm{LiTaO}_{3}$ due to the existence of a large internal field and the associated axial anisotropy in the polarization switching process. ${ }^{15}$ Improved domain poling techniques such as the nichrome $(\mathrm{NiCr})$ electrode $^{16}$ and backswitching of the poling wave form ${ }^{17}$ have been adapted to enhance the surface nucleation density

${ }^{a)}$ Electronic mail: peng@cc.ee.ntu.edu.tw and to control the domain relaxation in these widely used nonlinear materials.

In the electrical poling scheme, one takes advantage of current injection from the external circuit to compensate the internal and depolarization field so as to initiate the polarization switching process. ${ }^{17}$ Irregularity ${ }^{18}$ and broadening ${ }^{19}$ of inverted domains, however, have been constantly observed in the poling process and result in the loss of conversion efficiency. One such cause has been ascribed to the electrostatic interaction among the charged domain walls and nucleation steps. ${ }^{18}$ A more fundamental issue, however, can be related to tangential field $\left(E_{x}\right)$ enhanced current spreading in the unpatterned regime, which is normally coated with a layer of insulating material such as $\mathrm{SiO}_{2}$ (Ref. 20) or spin on glass. ${ }^{16}$

As illustrated in Fig. 1 of a conventional poling configuration, a relatively large $E_{x} \sim 4 E_{c}$ can exist underneath the insulating layer. Even though $E_{x}$ only affects a thin region up to $z \sim 0.25 \Lambda$ and fades away at larger distance, what is important is its field line pointing to an inward direction from both sides underneath the insulating layer. This phenomenon is believed to result in field screening and compensating in the unpatterned regime, and brings up the issue of domain

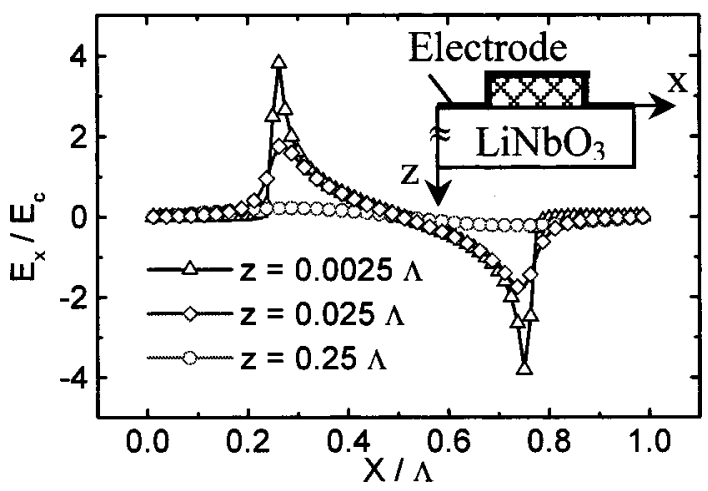

FIG. 1. Calculated tangential field $\left(E_{x}\right)$ distribution at various depths beneath the insulating layer. Inset shows a QPM device in a conventional poling configuration. 


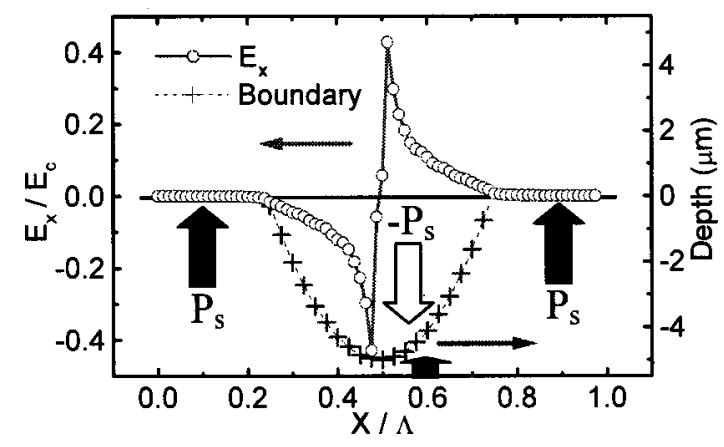

FIG. 2. Calculated tangential field $\left(E_{x}\right)$ at $z=0.0025 \Lambda$ underneath a positively charged parabola intervening between the electrode. The arrows indicate the polarity of $P_{s}$. Note the reduction of $E_{x}$ and outward field line with respect to those in Fig. 1.

broadening. ${ }^{21}$ Our calculations of $E_{x}$ and $E_{z}$ are based upon a finite-difference method capable of solving the Poisson equation with a spatial distribution of charge and dielectric. ${ }^{22}$ The structure parameters for evaluating the poling fields on the 500- $\mu \mathrm{m}$-thick congruent-grown $\mathrm{LiNbO}_{3}$ consist of an applied voltage of $10.5 \mathrm{kV}$, a dielectric constant of 30, a QPM periodicity $\Lambda$ of $20 \mu \mathrm{m}$, and an insulating layer of photoresist with $1 \mu \mathrm{m}$ thickness and dielectric constant of 3 , respectively.

In this work, we report a proof-of-concept mechanism in which we design a spatial distribution of $P_{s}$ in the subsurface regime to restrain the domain nucleation and motion in a subsequent field poling process. One such example is illustrated in Fig. 2, upon which a uniformly and positively charged parabola is introduced to represent the polarizationinduced charge in a head-to-head configuration of $P_{s}$. We thereby evaluate the $E_{x}$ at a depth $z=0.0025 \Lambda$ ( $\Lambda$ $=20 \mu \mathrm{m})$ along the parabolic domain boundary. As the induced charge density increases to $10^{-3} P_{s}$, we note the replacement of the insulating layer by the positively charged domain boundary not only can cause a substantial $E_{x}$ reduction (e.g., by one order of magnitude down to $0.4 E_{c}$ in the peak value), but also can result in a field line pointing to an outward direction. The latter signifies a promising mechanism to oppose the current spreading effect that would be valuable for electric poling.

We then proceeded with periodical polarization switching on Z-cut, 500- $\mu$ m-thick, double-side polished, congruent-grown undoped $\mathrm{LiNbO}_{3}$ substrates obtained from Crystal Technology. To derive a positive charge distribution via the divergence effect on $P_{s}$, we seek a solution by perturbing the subsurface domain structure with a polarity of $P_{s}$ opposite to that of the virgin crystal. ${ }^{23}$ A typical procedure is to let the patterned sample be placed inside a quartz tube furnace and undergo heat treatment at $1050^{\circ} \mathrm{C}$ for $5 \mathrm{~h}$ in an air ambience. The sample was then transferred to a poling apparatus consisting of a pulsed voltage amplifier made from Trek (model 20/20A for $20 \mathrm{kV}$ and $20 \mathrm{~mA}$ output). The poling setup was similar to that originally designed by Myers et al. ${ }^{24}$ in which a lithium chloride liquid electrode was made to contact the sample surfaces via a pair of viton $\mathrm{O}$ rings with $3 \mathrm{~mm}$ diam. To stabilize the domain reversal process, a fast turn-on rectifying diode was put in series with the poling apparatus such that relaxation of the inverted domain can be inhibited at termination of the pulsed field. ${ }^{25}$
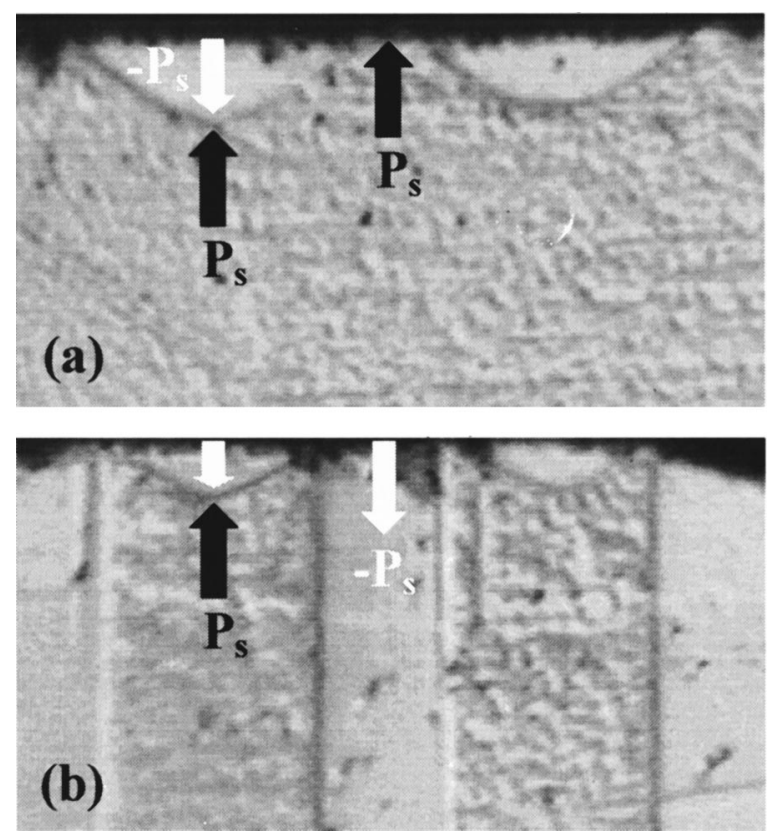

FIG. 3. $-y$ face micrograph of a PPLN-QPM device with $20 \mu \mathrm{m}$ period after (a) the first heat treatment at $1050{ }^{\circ} \mathrm{C}$ for $5 \mathrm{~h}$ and (b) followed up by a pulsed field poling at $21 \mathrm{kV} / \mathrm{mm}$. The substrate thickness is $500 \mu \mathrm{m}$.

Illustrated in Fig. 3(a) is the $-y$ face micrograph of an etched 20- $\mu \mathrm{m}$-period QPM structure after the first hightemperature treatment at $1050^{\circ} \mathrm{C}$. Albeit the thin $(\sim 50 \mathrm{~nm})$ $\mathrm{Al}$ electrode oxidizes and transforms into an $\mathrm{Al}_{2} \mathrm{O}_{3}$ pattern during the heat treatment, we note it can preserve the underlying $\mathrm{LiNbO}_{3}$ domain in its original polarization state. In addition, we find the porosity associated with microcracks in the $\mathrm{Al}_{2} \mathrm{O}_{3}$ electrode (not shown ${ }^{26}$ can provide a current limiting mechanism for the next step of pulsed field poling. Moreover, in the uncovered $\mathrm{LiNbO}_{3}$ regime, shallow surface domain inversion takes place in a triangular spatial distribution, whose thickness can be as deep as $0.25 \Lambda(5 \mu \mathrm{m})$. This phenomenon is in contrast to a recent study on $\mathrm{SiO}_{2}$-coated $\mathrm{LiNbO}_{3}$ (Ref. 27) and $\mathrm{KTP}^{28}$ upon which the stress-induced piezoelectric effect is reported to cause domain inversion under the $\mathrm{SiO}_{2}$ clad. These observations suggest that the origin of surface domain inversion in the present study is dominated by the space-charge effect arising from the outdiffusion and defect gradient associated with the hightemperature process. ${ }^{29-31}$

We further exhibit in Fig. 3(b) the etched $-y$ face micrograph of a periodically poled $\mathrm{LiNbO}_{3}$ QPM structure that has undergone both the heat treatment and pulsed field poling at $21 \mathrm{kV} / \mathrm{mm}$. Instead of resuming a forward domain growth along the existing $180^{\circ}$ triangular domain boundary, ${ }^{13}$ we now have the bulk periodical polarization switching nucleate under the oxidized $\mathrm{Al}_{2} \mathrm{O}_{3}$ electrode on the $+Z$ face. These observations confirm the use of micropores from the oxidized electrode to establish an electric contact and form nucleation sites to the underlying $\mathrm{LiNbO}_{3}$. In comparison, the inverted triangular domains formed during the first heat treatment remain unchanged and stay outside the electrode regime after the pulsed field poling.

The most important fact, however, is the observation of lateral $180^{\circ}$ domain motion confined to the edge of the triangular domain boundary. This phenomenon differs from a re- 

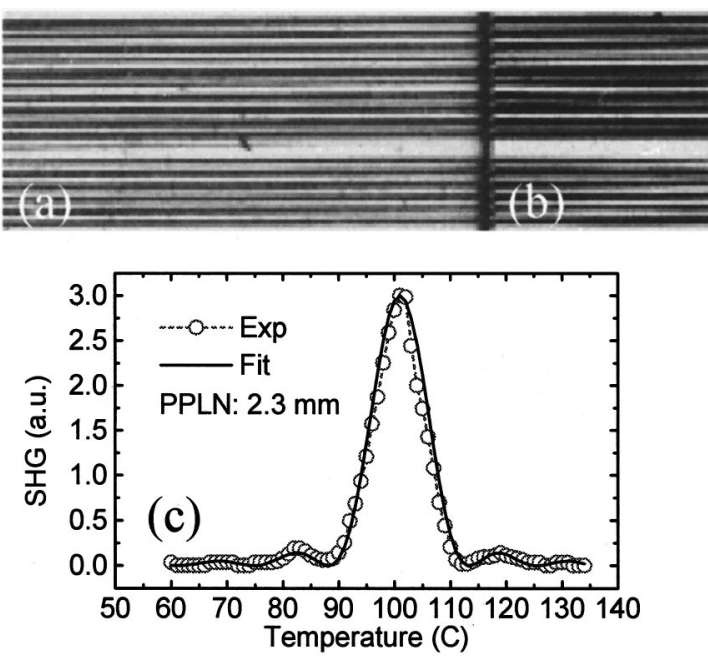

FIG. 4. Micrograph of a PPLN-QPM device with a nominal $6.7 \mu \mathrm{m}$ period in the (a) $-y$, and (b) $-Z$ faces, and (c) the temperature-tuning curves of the SHG power measurement and the fitting result. The substrate thickness is $500 \mu \mathrm{m}$.

cent investigation upon which a nonpolarized phase of cubic perovskite was introduced to suppress the sidewise domain motion in $\mathrm{LiTaO}_{3} .{ }^{6}$ The above observations suggest the positive charge, arising from the divergence of $P_{s}$ along the triangular domain boundary, can constitute a potential barrier against current spreading into the unpatterned regime and inhibit the propagation of domain forward growth along the existing boundary. In addition, it also supports the use of a repelling field line from the positive charge to reduce the tangential field and restrict the lateral $180^{\circ}$ domain motion upon field poling.

In extending this two-step poling procedure toward the realization of second-harmonic-generation (SHG) lasers in the green and blue spectral regimes, we illustrate in Fig. 4 the etched micrograph of the (a) $-y$, and (b) $-Z$ faces of a QPM device with a nominal $6.7 \mu \mathrm{m}$ period on $500-\mu \mathrm{m}$-thick $\mathrm{LiNbO}_{3}$. A close examination of the switched domains reveals a mirror image correspondence between the etched patterns in the $-y$ and $-Z$ faces, respectively. This observation confirms the forward growth of inverted domains and their restrictive motion bound by the positive charge. Using a cw $\mathrm{Nd}$ :YAG laser as the pumping source, data shown in Fig. 4(c) reveal a good temperature tuning curve for the SHG power measurement. The fitting result indicates the QPM device has reached a phase-matching length of $2.3 \mathrm{~mm}$, which is limited by the contact $\mathrm{O}$ ring used in the poling apparatus.

In summary, we report the use of electrostatic control via $-\nabla \cdot P_{s}=\rho$ to manipulate the interaction among domain walls and nucleation sites during the pulsed field poling process. By introducing a positive charge distribution intervening between the patterned electrodes, bulk periodical polar- ization switching in $\mathrm{LiNbO}_{3}$ is found to be confined to the edge of the charged domain boundary. These observations are ascribed to the constraint on domain nucleation and suppression of tangential field due to the polarization-induced charge at the inverted surface domain boundary.

The authors wish to acknowledge assistance from S.-M. Tsan, C.-C. Hsu, Professor W.-S. Wang, and the Integrated Optics and Solid State Laboratories at NTUEE. This research was supported by the National Science Council, Grant Nos. 89-2215-E-002-041 and 89-2215-E-002-047.

${ }^{1}$ J. A. Armstrong, N. Bloembergen, J. Ducuing, and P. S. Pershan, Phys. Rev. 127, 1918 (1962).

${ }^{2}$ M. M. Fejer, G. A. Magel, D. H. Jundt, and R. L. Byer, IEEE J. Quantum Electron. 28, 2631 (1992).

${ }^{3}$ L. E. Myers and W. R. Bosenberg, IEEE J. Quantum Electron. 33, 1663 (1997)

${ }^{4}$ A. V. Golenishchev-Kutuzov, V. A. Golenishchev-Kutuzov, and R. I. Kalimullin, Phys. Usp. 43, 647 (2000).

${ }^{5}$ Y. Yamada, N. Nada, M. Saitoh, and K. Watanabe, Appl. Phys. Lett. 62, 435 (1993).

${ }^{6}$ K. Mizuuchi and K. Yamamoto, Appl. Phys. Lett. 66, 2943 (1995).

${ }^{7}$ H. Karlsson, F. Laurell, and L. K. Cheng, Appl. Phys. Lett. 74, 1519 (1999).

${ }^{8}$ G. Rosenman, A. Skliar, Y. Findling, P. Urenski, A. Englander, P. A. Thomas, and Z. W. Hu, J. Phys. D 32, L49 (1999).

${ }^{9}$ Y. N. Korkishko, V. A. Fedorov, and F. Laurell, IEEE J. Sel. Top. Quantum Electron. 6, 132 (2000).

${ }^{10}$ Y. N. Korkishko, V. A. Fedorov, A. N. Alkaev, and F. Laurell, Appl. Phys. B: Lasers Opt. 73, 519 (2001).

${ }^{11}$ M. Katz, D. Eger, M. B. Oron, and A. Hardy, J. Appl. Phys. 90, 53 (2001).

${ }^{12}$ J. Camlibel, J. Appl. Phys. 40, 1690 (1969).

${ }^{13}$ R. C. Miller and G. Weinreich, Phys. Rev. 117, 1460 (1960).

${ }^{14}$ E. Fatuzzo and W. J. Merz, Phys. Rev. 116, 61 (1959).

${ }^{15}$ L.-H. Peng, Y.-C. Fang, and Y.-C. Lin, Appl. Phys. Lett. 74, 2070 (1999).

${ }^{16}$ G. D. Miller, R. G. Batchko, W. M. Tulloch, D. R. Weise, M. M. Fejer, and R. L. Byer, Opt. Lett. 22, 1834 (1997).

${ }^{17}$ R. G. Batchko, V. Y. Shur, M. M. Fejer, and R. L. Byer, Appl. Phys. Lett. 75, 1673 (1999).

${ }^{18}$ V. Y. Shur, E. L. Rumyantsev, E. V. Nikolaeva, and E. I. Shishkin, Appl. Phys. Lett. 77, 3636 (2000)

${ }^{19}$ P. Urenski, M. Molotskii, and G. Rosenman, Appl. Phys. Lett. 79, 2964 (2001).

${ }^{20}$ K. Mizuuchi, K. Yamamoto, and M. Kato, Appl. Phys. Lett. 70, 1201 (1997).

${ }^{21}$ G. Rosenman, Kh. Garb, A. Skliar, M. Oron, D. Eger, and M. Katz, Appl. Phys. Lett. 73, 865 (1998).

${ }^{22}$ L. Baudry and J. Tournier, J. Appl. Phys. 90, 1442 (2001).

${ }^{23}$ K. Nakamura, H. Ando, and H. Shimizu, Appl. Phys. Lett. 50, 1413 (1987).

${ }^{24}$ L. E. Myers, R. C. Eckardt, M. M. Fejer, R. L. Byer, W. R. Bosenberg, and J. W. Pierce, J. Opt. Soc. Am. B 12, 2102 (1995).

${ }^{25}$ L.-H. Peng, Y.-C. Zhang, and Y.-C. Lin, Appl. Phys. Lett. 78, 4 (2001).

${ }^{26}$ K. Shimizu, K. Kobayashi, G. E. Thompson, and G. C. Wood, Philos. Mag. A 66, 643 (1992).

${ }^{27}$ M. Fujimura, T. Suhara, and H. Nishihara, Electron. Lett. 27, 1207 (1991).

${ }^{28}$ K. S. Buritskii, E. M. Dianov, V. A. Maslov, V. A. Chernykh, and E. A. Shcherbakov, J. Appl. Phys. 79, 3345 (1996).

${ }^{29}$ J. Webjörn, F. Laurell, and G. Arvidsson, IEEE Photonics Technol. Lett. 1, 316 (1989).

${ }^{30}$ V. D. Kugel and G. Rosenman, Appl. Phys. Lett. 62, 2902 (1993).

${ }^{31}$ L. Huang and N. A. F. Jaeger, Appl. Phys. Lett. 65, 1763 (1994). 
Applied Physics Letters is copyrighted by the American Institute of Physics (AIP). Redistribution of journal material is subject to the AIP online journal license and/or AIP copyright. For more information, see http:/ojps.aip.org/aplo/aplcr.jsp

Copyright of Applied Physics Letters is the property of American Institute of Physics and its content may not be copied or emailed to multiple sites or posted to a listserv without the copyright holder's express written permission. However, users may print, download, or email articles for individual use. 